\title{
Analysis of Coupled System of Implicit Fractional Differential Equations Involving Katugampola-Caputo Fractional Derivative
}

\author{
Manzoor Ahmad, ${ }^{1}$ Jiqiang Jiang $\mathbb{D}^{2},{ }^{2}$ Akbar Zada $\mathbb{D}^{,},{ }^{1}$ Syed Omar Shah, ${ }^{3}$ and Jiafa Xu $\mathbb{D}^{2}$ \\ ${ }^{1}$ Department of Mathematics, University of Peshawar, Peshawar 25000, Pakistan \\ ${ }^{2}$ School of Mathematical Sciences, Qufu Normal University, Qufu 273165, China \\ ${ }^{3}$ Department of Physical and Numerical Sciences, Qurtuba University of Science and Information Technology, \\ Peshawar 25000, Pakistan \\ Correspondence should be addressed to Jiqiang Jiang; qfjjq@163.com
}

Received 9 December 2019; Accepted 13 March 2020; Published 21 April 2020

Academic Editor: Yan-Ling Wei

Copyright (c) 2020 Manzoor Ahmad et al. This is an open access article distributed under the Creative Commons Attribution License, which permits unrestricted use, distribution, and reproduction in any medium, provided the original work is properly cited.

In this paper, we study the existence and uniqueness of solutions to implicit the coupled fractional differential system with the Katugampola-Caputo fractional derivative. Different fixed-point theorems are used to acquire the required results. Moreover, we derive some sufficient conditions to guarantee that the solutions to our considered system are Hyers-Ulam stable. We also provided an example that explains our results.

\section{Introduction}

From the last few years, fractional differential equations $(\mathbb{E D E} \mathbb{E})$ theory has gained significant attraction and importance. It arises naturally in various models in areas such as control theory, biology, nonlinear waves of earthquake, mechanics, signal processing, modeling the seepage flow in porous media, and in fluid dynamics, memory mechanism and hereditary properties of materials. Some recent existence and uniqueness $(\mathbb{E} \mathbb{U})$ results of solutions for $\mathbb{E} \mathbb{E} \mathbb{E} \mathbb{S}$ with initial as well as boundary conditions can be found in $[1-8]$. In fact, $\mathbb{E} \mathbb{D} \mathbb{E} \mathbb{S}$ are the effective tools in real-world problems that motivate many researchers to work in this field, see [6, 9-20] and references cited therein.

Another important aspect in the qualitative theory of differential equations $(\mathbb{D E} \mathbb{E})$, which is exclusively studied for

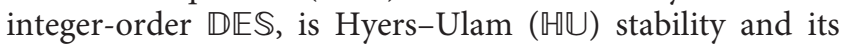
various types. This stability concept was originated in 1940 from the question of Ulam [21], which was answered by Hyers [22]. Many researchers extended and generalized Hyers's results in which the work of Rassias [23] is considered to be the first notable contribution. Many researchers studied $\mathbb{U} \mathbb{U}$ and $\mathbb{U} U$-Rassias stability of various functional equations, see [24-44] and references cited therein. This field got notorious attention when mathematicians started studying the $\mathbb{Q U}$ stability for the solution of differential equations, initiated by Obloza [45, 46]. Motivated by the work of Obloza, various classes of integer-order ordinary differential equations were investigated [39, 47]. The idea was then extended for nonintegral-order differential equations; for some recent work, we refer to [48, 49]. As far as we know, only few researchers studied the different kinds of Ulam's type stabilities for the coupled system of $\mathbb{E} \mathbb{D} \mathbb{E} S$. For details, see $[38,50-52]$.

Nowadays, both Riemann-Liouville-type $(\mathbb{R L})$ and Caputo-type derivatives are introduced generally, and the impact of applying it in mathematical physics and equations associated with probability is exposed. The fractional integral that generalizes both $\mathbb{R} \mathbb{L}$ - and Hadamard-type integrals into a single form was initiated by Katugampola [53]. Later on, in [54], new fractional derivative that generalizes the two derivatives was introduced by Katugampola.

Motivated by the work $[54,55]$, in this paper, we study the $\mathbb{E} \mathbb{U}$ and $\mathbb{U} \mathbb{U}$ stability of the following implicit switched coupled system of $\mathbb{E D E}$ involving the Katugampola-Caputo $(\mathbb{K C})$ fractional derivative: 


$$
\left\{\begin{array}{l}
{ }_{c}^{\sigma} D^{\alpha} u(\sigma)-f\left(\varsigma, u(\sigma),{ }_{c}^{\sigma} D^{\alpha} u(\sigma)\right)=\psi(\sigma, u(\sigma), v(\sigma)), \\
\sigma \in \mathbf{J}=[0, T], T>0, \quad 0<\alpha<1, \\
{ }_{c}^{\sigma} D^{\alpha} v(\sigma)-g\left(\varsigma, v(\sigma),{ }_{c}^{\sigma} D^{\alpha} v(\sigma)\right)=\varphi(\sigma, u(\sigma), v(\sigma)), \\
u(0)+h(u)=u_{0}, v(0)+h(v)=v_{0},
\end{array}\right.
$$

where $\sigma$ is a positive real number and ${ }_{c}^{\sigma} D^{\alpha} u(\sigma)$ and ${ }_{c}^{\sigma} D^{\alpha} v(\sigma)$ are the Katugampola-Caputo fractional derivatives of $u(\sigma)$ and $v(\sigma)$, respectively. The functions $f, g: \mathbf{J} \times$ $\mathbb{X} \times \mathbb{X} \times \longrightarrow \mathbb{X}$ are closed and bounded. Also, $\psi$ and $\varphi$ are nonlocal continuous functions.

\section{Preliminaries}

In this portion, we introduce some notions and preliminaries. Suppose $\mathbb{C}(\mathbf{J}, \mathbb{X})$ denotes the Banach space $(\mathbb{B S})$ of continuous functions from $\mathbf{J}=[0, T]$ into $\mathbb{X}$, defined by $\mathbb{C}(\mathbf{J}, \mathbb{X})=\{u: \mathbf{J} \longrightarrow \mathbb{X}, \sigma \in \mathbf{J}\}$, endowed with norms as $\|u\|=\max _{\sigma \in \mathrm{J}}\|u(\sigma)\|,\|v\|=\max _{\sigma \in \mathrm{J}}\|v(\sigma)\|$; indeed, these are $\mathbb{B S s}$ ' under these norms, and hence, their product is also $\mathbb{B S}$ with $\|(u, v)\|=\|u\|+\|v\|$, where $u$ and $v$ are in $\mathbb{C}(\mathbf{J}, \mathbb{X})$.

Definition 1 (see [5]). Let $\alpha>0$, and the arbitrary order integral in the $\mathbb{R} \mathbb{L}$ sense for a function $p: \mathbf{J} \longrightarrow \mathbb{R}$ is

$$
\square^{\alpha} p(\sigma)=\frac{1}{\Gamma(\alpha)} \int_{0}^{\sigma}(\sigma-v)^{\alpha-1} p(v) \mathrm{d} v,
$$

where the integral on the right-hand side (RHS) is pointwise defined on $\mathbb{R}^{+}$.

Definition 2 (see [54]). $\mathbb{K C}$ left-sided noninteger-order integral ${ }^{\rho} I_{a^{+}}^{\alpha} f$ of the function $f$ on a closed interval $[a, b]$ of order $\alpha$ is defined as

$$
\left({ }^{\rho} \mathrm{I}^{\alpha} f\right)(\sigma)=\frac{\rho^{1-\alpha}}{\Gamma(\alpha)} \int_{a}^{\sigma}\left(\sigma^{\rho}-v^{\rho}\right)^{\alpha-1} v^{\rho-1} \mathrm{~d} v
$$

where $\alpha \in C$, with $\mathfrak{R}(\alpha)>0$.

The corresponding $\mathbb{K} \mathbb{C}$ fractional derivative to the above integral is given by

$$
\left({ }_{c}^{\rho} D_{a^{+}}^{\alpha} f\right)(\sigma)=\frac{\rho^{\alpha-n+1}}{\Gamma(n-1)}\left(\sigma^{1-\rho} \frac{\mathrm{d}}{\mathrm{d} t}\right)^{n} \int_{a}^{\sigma}\left(\sigma^{\rho}-v^{\rho}\right)^{n-\alpha-1} f(v) \mathrm{d} v .
$$

Definition 3 (see [5]). The noninteger-order derivative in the Caputo sense of $p$ on closed interval $[a, b]$ is

$$
\frac{\mathrm{d}^{\alpha}}{\mathrm{d} \sigma^{\alpha}} p(\sigma)=\int_{a}^{\sigma} \frac{(\sigma-v)^{n-\alpha-1}}{\Gamma(n-\alpha)}\left(\frac{d^{n}}{d v^{n}} p(v)\right) \mathrm{d} v, \quad \alpha \in(n-1, n],
$$

where $n-1=[\alpha]$. In particular,

$$
\begin{aligned}
\frac{\mathrm{d}^{\alpha}}{\mathrm{d} \sigma^{\alpha}} p(\sigma) & =\frac{1}{\Gamma(1-\alpha)} \int_{a}^{\sigma} \frac{p^{\prime}(v)}{(\sigma-v)^{\alpha}} \mathrm{d} v, \\
\text { where } \varphi^{\prime}(v) & =\frac{\mathrm{d} \varphi(v)}{\mathrm{d} v}, \alpha \in(0,1] .
\end{aligned}
$$

Moreover, the integral on the RHS is pointwise defined on $\mathbb{R}^{+}$.

Lemma 1 (see [56]). Let $\alpha \in[n-1, n)$, for $p \in \mathbb{C}([a, b])$, and the only one solution of $\left(d^{\alpha} / d \sigma^{\alpha}\right) p(\sigma)=0$ has the formula $p(\sigma)=\sum_{k=0}^{[\alpha]} c_{k} \sigma^{k}$, where $c_{k} \in \mathbb{R}, k=1,2, \ldots,[\alpha]$, $[\alpha]=n-1$.

Lemma 2 (see [56]). Let $\alpha \in[n-1, n)$, for $p \in \mathbb{C}([a, b])$ and $\square^{\alpha}\left(d^{\alpha} / d \sigma^{\alpha}\right) p(\sigma)=p(\sigma)+\sum_{k=0}^{[\alpha]} a_{k} \sigma^{k}, \quad$ for some $a_{k} \in \mathbb{R}$, $k=1,2, \ldots,[\alpha],[\alpha]=n-1$.

Definition 4 (see [57]). Consider $\mathbb{B S} \mathbb{X}$. Let $\Omega_{1}, \Omega_{2}: \mathbb{X} \times$ $\mathbb{X} \longrightarrow \mathbb{X}$ be two operators, and the operator system

$$
\left\{\begin{array}{l}
p(\sigma)=\Omega_{1}(p, q)(\sigma) \\
q(\sigma)=\Omega_{2}(p, q)(\sigma)
\end{array}\right.
$$

is called $\mathbb{U}$ stable if we can find constants $C_{i}(i=1,2,3,4)>0$ such that, for each $\varrho_{j}(j=1,2)>0$ and for each solution $(\widehat{p}, \widehat{q}) \in \mathbb{X} \times \mathbb{X}$ of the inequalities

$$
\left\{\begin{array}{l}
\|\widehat{p}-\psi(\widehat{p}, \widehat{q})\| \leq \varrho_{1} \\
\|\widehat{q}-\varphi(\widehat{p}, \widehat{q})\| \leq \varrho_{2}
\end{array}\right.
$$

hold, then there exists a solution $(\widetilde{p}, \widetilde{q}) \in \mathbb{X}$ of system $(7)$, which satisfies the inequalities

$$
\left\{\begin{array}{l}
\|\widehat{p}-\tilde{p}\| \leq C_{1} \varrho_{1}+C_{2} \varrho_{2} \\
\|\widehat{q}-\tilde{q}\| \leq C_{3} \varrho_{1}+C_{4} \varrho_{2} .
\end{array}\right.
$$

Lemma 3 (see [57], Theorem 4). Consider $\mathbb{B S} \mathbb{X}$ with operators $\Omega_{1}, \Omega_{2}: \mathbb{X} \times \mathbb{X} \longrightarrow \mathbb{X}$ such that

$$
\left\{\begin{array}{l}
\left\|\Omega_{1}(p, q)-\Omega_{1}(\widehat{p}, \hat{q})\right\| \leq \Lambda_{1}\|p-\widehat{p}\|+\Lambda_{2}\|q-\hat{q}\|, \\
\left\|\Omega_{2}(p, q)-\Omega_{2}(\widehat{p}, \hat{q})\right\| \leq \Lambda_{3}\|p-\widehat{p}\|+\Lambda_{4}\|q-\hat{q}\| .
\end{array}\right.
$$

If the spectral radius of

$$
\mathbb{H}=\left(\begin{array}{cc}
\Lambda_{1} & \Lambda_{2} \\
\Lambda_{3} & \Lambda_{4}
\end{array}\right)
$$

is less than one, then the fixed points corresponding to operational system (10) are ㅁ stable.

\section{Existence and Uniqueness of the Solution}

In this section, we prove $\mathbb{E} \mathbb{U}$ of system (1). We consider the following assumptions:

$\left(\mathbb{E}_{1}\right)$ : suppose $\psi, \varphi: \mathbf{J} \times \mathbb{X} \times \mathbb{X} \longrightarrow \mathbb{X}$ are continuous, and for all $(\mu, \nu),(\bar{\mu}, \bar{\nu}) \in \mathbb{X} \times \mathbb{X}$ with $\sigma \in \mathbf{J}$, there exist $\mathbb{M}_{\psi}, \mathbb{M}_{\varphi}, \mathbb{M}_{\psi}^{\prime}, \mathbb{M}_{\varphi}^{\prime}>0$ such that 


$$
\begin{aligned}
& \|\psi(\sigma, \mu(\sigma), \nu(\sigma))-\psi(\sigma, \bar{\mu}(\sigma), \bar{\nu}(\sigma))\| \leq \mathbb{M}_{\psi}\|\mu(\sigma)-\bar{\mu}(\sigma)\| \\
& \quad+\mathbb{M}_{\psi}^{\prime}\|\nu(\sigma)-\bar{\nu}(\sigma)\|, \\
& \|\varphi(\sigma, \mu(\sigma), \nu(\sigma))-\varphi(\sigma, \bar{\mu}(\sigma), \bar{\nu}(\sigma))\| \leq \mathbb{M}_{\varphi}\|\mu(\sigma)-\bar{\mu}(\sigma)\| \\
& \quad+\mathbb{M}_{\varphi}^{\prime}\|\nu(\sigma)-\bar{\nu}(\sigma)\| .
\end{aligned}
$$

$\left(\mathbb{Z}_{2}\right)$ : there exist $\mathbb{L}_{f}, \mathbb{L}_{g}, \mathbb{L}_{f}^{\prime}, \mathbb{L}_{g}^{\prime}>0$ such that

$$
\begin{aligned}
& \|f(\sigma, \mu(\sigma), \nu(\sigma))-f(\sigma, \bar{\mu}(\sigma), \bar{\nu}(\sigma))\| \leq \mathbb{L}_{f}\|\mu-\bar{\mu}\| \\
& \quad+\mathbb{L}_{f}^{\prime}\|\nu-\bar{\nu}\|, \\
& \|g(\sigma, \mu(\sigma), \nu(\sigma))-g(\sigma, \bar{\mu}(\sigma), \bar{\nu}(\sigma))\| \leq \mathbb{L}_{g}\|\mu-\bar{\mu}\| \\
& \quad+\mathbb{L}_{g}^{\prime}\|\nu-\bar{\nu}\|,
\end{aligned}
$$

for all $\sigma \in \mathbf{J},(\mu, \nu),(\bar{\mu}, \bar{\nu}) \in \mathbb{X} \times \mathbb{X}$.

$\left(\mathbb{H}_{3}\right)$ : suppose $h: C(\mathbf{J}, \mathbb{X}) \longrightarrow \mathbb{R}$ is continuous, and for all $\mu, v, \bar{\mu}, \bar{v} \in \mathbb{X}$, there exist $a, b>0$ such that

$\|h(\mu)-h(\bar{\mu})\| \leq a\|\mu-\bar{\mu}\|$ and $\|h(\nu)-h(\bar{\nu})\| \leq b\|\nu-\bar{\nu}\|$.

$\left(\mathbb{E}_{4}\right)$ : let $l_{f}, l_{g}, p, q, p^{*}, q^{*} \in C(\mathbf{J}, \mathbb{X})$ with $l_{g h}=\sup$ $\left\{\left|l_{f}(\sigma), l_{g}(\sigma)\right|\right\}<1$ such that $\|f(\sigma, \mu(\sigma), v(\sigma))\| \leq l_{f}(\sigma)+p(\sigma)\|\mu\|_{P C}+q(\sigma)\|\nu\|$,

$\|g(\sigma, \mu(\sigma), \nu(\sigma))\| \leq l_{g}(\sigma)+p^{*}(\sigma)\|\mu\|_{P C}+q^{*}(\sigma)\|\nu\|$,

where $\sigma \in \mathbf{J}$.

$\left(\mathbb{H}_{5}\right)$ : let $\psi, \varphi: \mathbf{J} \times \mathbb{X} \times \mathbb{X} \longrightarrow \mathbb{X}$ be continuous, and for all $(\mu, \nu) \in \mathbb{X} \quad$ with $\quad \varsigma \in \mathbf{J}$, there exist $l_{\psi}, l_{\psi}^{1}, l_{\psi}^{2}, l_{\varphi}, l_{\varphi}^{1}, l_{\varphi}^{2} \in C(\mathbf{J}, \mathbb{X})$ such that

$$
\begin{gathered}
\|\psi(\sigma, \mu(\sigma), \nu(\sigma))\| \leq l_{\psi}(\sigma)+l_{\psi}^{1}(\sigma)\|\mu(\sigma)\|+l_{\psi}^{2}\|\nu(\sigma)\|, \\
\|\varphi(\sigma, \mu(\sigma), \nu(\sigma))\| \leq l_{\varphi}(\sigma)+l_{\varphi}^{1}(\sigma)\|\mu(\sigma)\|+l_{\varphi}^{2}\|\nu(\sigma)\| .
\end{gathered}
$$

Theorem 1. Let $\theta_{1}, \theta_{2} \in C(\mathbf{J}, \mathbb{X})$ and $f, g: J \times \mathbb{X} \times \mathbb{X} \longrightarrow \mathbb{X}$ be continuous, and the solution of

$$
\left\{\begin{array}{l}
{ }_{c}^{\sigma} D^{\alpha} \mu(\sigma)-f\left(\varsigma, \mu(\sigma),{ }_{c}^{\sigma} D^{\alpha} \mu(\sigma)\right)=\theta_{1}(\sigma), \\
{ }_{c}^{\sigma} D^{\alpha} v(\sigma)-f\left(\varsigma, v(\sigma),{ }_{c}^{\sigma} D^{\alpha} v(\sigma)\right)=\theta_{2}(\sigma), \\
\mu(0)+h(\mu)=\mu_{0}, v(0)+h(v)=v_{0},
\end{array}\right.
$$

is equivalent to

$$
\left\{\begin{array}{l}
\mu(\sigma)=\mu_{0}-h(\mu)+\frac{\sigma^{1-\alpha}}{\Gamma(\alpha)} \int_{0}^{\sigma}\left(\sigma^{\sigma}-v^{\sigma}\right)^{\alpha-1} v^{\sigma-1} f\left(v, \mu(v),{ }_{c}^{\sigma} D^{\alpha} \mu(v)\right) \mathrm{d} v+\frac{\sigma^{1-\alpha}}{\Gamma(\alpha)} \int_{0}^{\sigma}\left(\sigma^{\sigma}-v^{\sigma}\right)^{\alpha-1} v^{\sigma-1} \theta_{1}(v) \mathrm{d} v, \\
v(\sigma)=v_{0}-h(v)+\frac{\sigma^{1-\alpha}}{\Gamma(\alpha)} \int_{0}^{\sigma}\left(\sigma^{\sigma}-v^{\sigma}\right)^{\alpha-1} v^{\sigma-1} g\left(v, v(v),{ }_{c}^{\sigma} D^{\alpha} \nu(v)\right) \mathrm{d} v+\frac{\sigma^{1-\alpha}}{\Gamma(\alpha)} \int_{0}^{\sigma}\left(\sigma^{\sigma}-v^{\sigma}\right)^{\alpha-1} v^{\sigma-1} \theta_{2}(v) \mathrm{d} v .
\end{array}\right.
$$

\section{Proof. Consider}

${ }_{c}^{\sigma} D^{\alpha} \mu(\sigma)-f\left(\sigma, \mu(\sigma),{ }_{c}^{\sigma} D^{\alpha} \mu(\sigma)\right)=\theta_{1}(\sigma), \quad 0<\alpha<1, \sigma \in \mathbf{J}$.

Applying integral ${ }^{\rho} I^{\alpha}$, we get

$$
\begin{aligned}
{ }^{\sigma} I^{\alpha} & \left({ }_{c}^{\sigma} D^{\alpha} \mu(\sigma)\right)-{ }^{\rho} I^{\alpha}\left(f\left(\sigma, \mu(\sigma),{ }_{c}^{\sigma} D^{\alpha} \mu(\sigma)\right)\right) \\
= & { }^{\rho} \mathrm{I}^{\alpha}\left(\theta_{1}(\sigma)\right)-c_{0} \\
\mu(\sigma)= & \frac{\sigma^{1-\alpha}}{\Gamma(\alpha)} \int_{0}^{\sigma}\left(\sigma^{\sigma}-v^{\sigma}\right)^{\alpha-1} v^{\sigma-1} f\left(v, \mu(v),{ }_{c}^{\sigma} D^{\alpha} \mu(v)\right) \mathrm{d} v \\
& +\frac{\sigma^{1-\alpha}}{\Gamma(\alpha)} \int_{0}^{\sigma}\left(\sigma^{\sigma}-v^{\sigma}\right)^{\alpha-1} v^{\sigma-1} \theta_{1}(v) \mathrm{d} v-c_{0} .
\end{aligned}
$$

Using $\mu(0)+h(\mu)=\mu_{0}$, we have

$$
\begin{aligned}
\mu(\sigma)= & \mu_{0}-h(\mu)+\frac{\sigma^{1-\alpha}}{\Gamma(\alpha)} \int_{0}^{\sigma}\left(\sigma^{\sigma}-v^{\sigma}\right)^{\alpha-1} v^{\sigma-1} \\
& \cdot f\left(v, \mu(v),{ }_{c}^{\sigma} D^{\alpha} \mu(v)\right) \mathrm{d} v \\
& +\frac{\sigma^{1-\alpha}}{\Gamma(\alpha)} \int_{0}^{\sigma}\left(\sigma^{\sigma}-v^{\sigma}\right)^{\alpha-1} v^{\sigma-1} \theta_{1}(v) \mathrm{d} v .
\end{aligned}
$$

Similarly,

$$
\begin{aligned}
\nu(\sigma)= & v_{0}-h(v)+\frac{\sigma^{1-\alpha}}{\Gamma(\alpha)} \int_{0}^{\sigma}\left(\sigma^{\sigma}-v^{\sigma}\right)^{\alpha-1} v^{\sigma-1} \\
& \cdot g\left(v, \nu(v),{ }_{c}^{\sigma} D^{\alpha} \nu(v)\right) \mathrm{d} v \\
& +\frac{\sigma^{1-\alpha}}{\Gamma(\alpha)} \int_{0}^{\sigma}\left(\sigma^{\sigma}-v^{\sigma}\right)^{\alpha-1} v^{\sigma-1} \theta_{2}(v) \mathrm{d} v .
\end{aligned}
$$

For the upcoming result, here, we define an operator.

$\left(\mathbb{U}_{6}\right)$ : choose $\mathbb{B}_{r}=\{(\mu, \nu) \in \mathbb{X} \times \mathbb{X}:\|(\mu, \nu)\| \leq r,\|u\| \leq$ $(r / 2),\|\nu\| \leq(r / 2)\} \subset \mathbb{X}$, with $r \geq\left(\left(\mu_{1}+\left\|\mu_{0}\right\|+\left\|\nu_{0}\right\|+H+G\right) /\right.$ $\left(\left(1-\mu_{2}\right)\right), \quad p=\sup \{p(\sigma): \sigma \in \mathbf{J}\}, \quad q=\sup \{q(\sigma): \sigma \in \mathbf{J}\}$, $H=\max \{\|h(\mu)\|, \mu \in \mathbb{X}\}, G=\max \{\|h(\nu)\|, \nu \in \mathbb{X}\}, l_{\psi}=\sup$ $\left\{l_{\psi}(\sigma): \sigma \in \mathbf{J}\right\}, \quad l_{\psi}^{1}=\sup \left\{l_{\psi}^{1}(\sigma): \sigma \in \mathbf{J}\right\}, \quad$ and $\quad l_{\psi}^{2}=\sup$ $\left\{l_{\psi}^{2}(\sigma): \sigma \in \mathbf{J}\right\}$. 


$$
\begin{aligned}
& \mu_{1}=\frac{T^{\sigma \alpha} l_{g h}\left(2-\left(q+q^{*}\right)+(1-q)\left(1-q^{*}\right)\left(l_{\psi}+l_{\varphi}\right)\right)}{\sigma^{\alpha}(1-q)\left(1-q^{*}\right) \Gamma(\alpha+1)}, \\
& \mu_{2}=\frac{T^{\sigma \alpha}\left(\left(1-q^{*}\right) p+(1-q) p^{*}+(1-q)\left(1-q^{*}\left(l_{\psi}^{1}+l_{\varphi}^{1}+l_{\psi}^{2}+l_{\varphi}^{2}\right)\right)\right)}{2 \sigma^{\alpha}(1-q)\left(1-q^{*}\right) \Gamma(\alpha+1)},
\end{aligned}
$$

$\max \left\{\mu_{1}, \mu_{2}\right\}<1$.

Construct $\mathbb{F}=\left(\mathbb{F}_{1}, \mathbb{F}_{2}\right)$ and $\mathbb{G}=\left(\mathbb{G}_{1}, \mathbb{G}_{2}\right)$ on $\mathbb{B}_{r}$ as

$$
\begin{aligned}
& \left\{\begin{array}{l}
\left(\mathbb{F}_{1} \mu\right)(\sigma)=\mu_{0}-h(\mu)+\frac{\sigma^{1-\alpha}}{\Gamma(\alpha)} \int_{0}^{\sigma}\left(\sigma^{\sigma}-v^{\sigma}\right)^{\alpha-1} v^{\sigma-1} f\left(v, \mu(v),{ }_{c}^{\sigma} D^{\alpha} \mu(v)\right) \mathrm{d} v, \\
\left(\mathbb{F}_{2} \nu\right)(\sigma)=v_{0}-h(\nu)+\frac{\sigma^{1-\alpha}}{\Gamma(\alpha)} \int_{0}^{\sigma}\left(\sigma^{\sigma}-v^{\sigma}\right)^{\alpha-1} v^{\sigma-1} g\left(v, \mu(v),{ }_{c}^{\sigma} D^{\alpha} \mu(v)\right) \mathrm{d} v,
\end{array}\right. \\
& \left\{\begin{array}{l}
\left(\mathbb{G}_{1} \mu\right)(\sigma)=\frac{\sigma^{1-\alpha}}{\Gamma(\alpha)} \int_{0}^{\sigma}\left(\sigma^{\sigma}-v^{\sigma}\right)^{\alpha-1} v^{\sigma-1} \psi(v, \mu(v), v(v)) \mathrm{d} v, \\
\left(\mathbb{G}_{2} v\right)(\sigma)=\frac{\sigma^{1-\alpha}}{\Gamma(\alpha)} \int_{0}^{\sigma}\left(\sigma^{\sigma}-v^{\sigma}\right)^{\alpha-1} v^{\sigma-1} \varphi(v, \mu(v), v(v)) \mathrm{d} v .
\end{array}\right.
\end{aligned}
$$

Theorem 2. Let the conditions from $\left(\mathbb{H}_{1}\right)$ to $\left(\mathbb{H}_{6}\right)$ hold; then, system (1) has only one solution.

Proof. For any $(\mu, \nu) \in \mathbb{B}_{r}$,

$$
\begin{gathered}
\|\mathbb{F}(\mu, v)+\mathbb{G}(\mu, v)\| \leq\|\mathbb{F}(\mu, v)\|+\|\mathbb{G}(\mu, v)\| \\
=\left\|\mathbb{F}_{1} \mu\right\|+\left\|\mathbb{F}_{2} v\right\|+\left\|\mathbb{G}_{1}(\mu, \nu)\right\|+\left\|\mathbb{G}_{2}(\mu, \nu)\right\| .
\end{gathered}
$$

From (24), we have

$$
\begin{aligned}
\left\|\mathbb{F}_{1} \mu(\sigma)\right\| & =\left|\mu_{0}-h(\mu)+\frac{\sigma^{1-\alpha}}{\Gamma(\alpha)} \int_{0}^{\sigma}\left(\sigma^{\sigma}-v^{\sigma}\right)^{\alpha-1} v^{\sigma-1} f\left(v, \mu(v),{ }_{c}^{\sigma} D^{\alpha} \mu(v)\right) \mathrm{d} v\right| \\
& \leq\left\|\mu_{0}-h(\mu)\right\|+\frac{\sigma^{1-\alpha}}{\Gamma(\alpha)} \int_{0}^{\sigma}\left(\sigma^{\sigma}-v^{\sigma}\right)^{\alpha-1} v^{\sigma-1}\left\|f\left(v, \mu(v),{ }_{c}^{\sigma} D^{\alpha} \mu(v)\right)\right\| \mathrm{d} v \\
& \leq\left\|\mu_{0}\right\|+\|h(\mu)\|+\frac{\sigma^{1-\alpha}}{\Gamma(\alpha)} \int_{0}^{\varsigma}\left(\sigma^{\sigma}-v^{\sigma}\right)^{\alpha-1} v^{\sigma-1}\left\|K_{\mu}(v)\right\| \mathrm{d} v \text {, where } \\
\left\|K_{\mu}(\sigma)\right\| & =\left\|f\left(\varsigma, \mu(\sigma),{ }_{c}^{\sigma} D^{\alpha} \mu(\sigma)\right)\right\|=\left\|f\left(\sigma, \mu_{0}-h(\mu)+{ }_{c}^{\sigma} I^{\alpha} K_{\mu}(\sigma), K_{\mu}(\sigma)\right)\right\| \\
& \leq l_{g h}+p(\sigma)\|\mu\|+q(\sigma)\left\|K_{\mu}(\sigma)\right\| \\
& \leq l_{g h}+p\|\mu\|+q\left\|K_{\mu}(\sigma)\right\|, \text { or } \\
\left\|K_{\mu}(\sigma)\right\| & \leq \frac{l_{g h}+p\|\mu\|}{1-q} .
\end{aligned}
$$


Therefore,

$$
\text { Furthermore, (24) gives }
$$

$$
\begin{aligned}
& \left\|\mathbb{F}_{1} \mu\right\| \leq\left\|\mu_{0}\right\|+H+\frac{\left(2 l_{g h}+r p\right) T^{\sigma \alpha}}{2(1-q) \sigma^{\alpha} \Gamma(\alpha+1)}, \\
& \left\|\mathbb{F}_{2} \mu\right\| \leq\left\|v_{0}\right\|+G+\frac{\left(2 l_{g h}+r p^{*}\right) T^{\sigma \alpha}}{2\left(1-q^{*}\right) \sigma^{\alpha} \Gamma(\alpha+1)} .
\end{aligned}
$$

$$
\begin{aligned}
\left\|\left(\mathbb{G}_{1}(\mu, v)\right)(\sigma)\right\| & =\left\|\frac{\sigma^{1-\alpha}}{\Gamma(\alpha)} \int_{0}^{\sigma}\left(\sigma^{\sigma}-v^{\sigma}\right)^{\alpha-1} v^{\sigma-1} \psi(v, \mu(v), v(v)) \mathrm{d} v\right\| \\
& \leq \frac{\sigma^{1-\alpha}}{\Gamma(\alpha)} \int_{0}^{\sigma}\left(\sigma^{\sigma}-v^{\sigma}\right)^{\alpha-1} v^{\sigma-1}\|\psi(v, \mu(v), v(v))\| \mathrm{d} v \\
& \leq \frac{\sigma^{1-\alpha}}{\Gamma(\alpha)} \int_{0}^{\sigma}\left(\sigma^{\sigma}-v^{\sigma}\right)^{\alpha-1} v^{\sigma-1} l_{\psi}(\sigma)+l_{\psi}^{1}(\sigma)\|\mu(\sigma)\|+l_{\psi}^{2}(\sigma)\|v(\sigma)\| \mathrm{d} v \\
& \leq \frac{T^{\sigma \alpha}\left(l_{\psi}(\sigma)+l_{\psi}^{1}(\sigma)\|\mu(\sigma)\|+l_{\psi}^{2}(\sigma)\|v(\sigma)\|\right)}{\sigma^{\alpha} \Gamma(\alpha+1)} \\
& \leq \frac{T^{\sigma \alpha}\left(l_{\psi}+l_{\psi}^{1}\|\mu\|+l_{\psi}^{2}\|v\|\right)}{\sigma^{\alpha} \Gamma(\alpha+1)} \\
& \leq \frac{T^{\sigma \alpha}\left(2 l_{\psi}+r\left(l_{\psi}^{1}+l_{\psi}^{2}\right)\right)}{2 \sigma^{\alpha} \Gamma(\alpha+1)} .
\end{aligned}
$$

In a similar way, we get

$$
\left\|\left(\mathbb{G}_{2}(\mu, \nu)\right)\right\| \leq \frac{T^{\sigma \alpha}\left(2 l_{\varphi}+r\left(l_{\varphi}^{1}+l_{\psi}^{2}\right)\right)}{2 \sigma^{\alpha} \Gamma(\alpha+1)} .
$$

$\|\mathbb{F}(\mu, \nu)+\mathbb{G}(\mu, \nu)\| \leq r$.
So, $\mathbb{F}(\mu, \nu)+\mathbb{G}(\mu, \nu) \in \mathbb{B}_{r}$.

Now, for $(\mu, \nu),(\bar{\mu}, \bar{\nu}) \in \mathbb{B}_{r}$ and $\varsigma \in \mathbf{J}$, we have

Therefore, (25) gives

$$
\begin{aligned}
& \|\mathbb{F}(\mu, \nu)-\mathbb{F}(\bar{\mu}, \bar{\nu})\| \leq\left\|\mathbb{F}_{1}(\mu)-\mathbb{F}_{1}(\bar{\mu})\right\|+\left\|\mathbb{F}_{2}(\nu)-\mathbb{F}_{1}(\bar{\nu})\right\| \\
& +\|h(\nu)-h(\bar{\nu})\|+\frac{\sigma^{\alpha-1}}{\Gamma(\alpha)} \int_{0}^{\sigma}\left(\sigma^{\sigma}-v^{\sigma}\right)^{\alpha-1} v^{\sigma-1}\left\|K_{\mu}(v)-K_{\bar{\mu}}(v)\right\| \mathrm{d} v \\
& K_{\mu}(v)=f\left(v, \mu(v),{ }_{c}^{\sigma} D^{\alpha} \mu(v)\right), \\
& K_{\bar{\mu}}(v)=f\left(v, \bar{\mu}(v),{ }_{c}^{\sigma} D^{\alpha} \bar{\mu}(v)\right), \\
& K_{v}(v)=g\left(v, \mu(v),{ }_{c}^{\sigma} D^{\alpha} \mu(v)\right), \\
& K_{\bar{\nu}}(v)=g\left(v, \bar{\mu}(v),{ }_{c}^{\sigma} D^{\alpha} \mu(v)\right) .
\end{aligned}
$$$$
\leq\|h(\mu)-h(\bar{\mu})\|+\frac{\sigma^{\alpha-1}}{\Gamma(\alpha)} \int_{0}^{\sigma}\left(\sigma^{\sigma}-v^{\sigma}\right)^{\alpha-1} v^{\sigma-1}\left\|K_{\mu}(v)-K_{\bar{\mu}}(v)\right\| \mathrm{d} v
$$$$
\left\|K_{\mu}(\sigma)-K_{\bar{\mu}}(\sigma)\right\| \leq\left\|f\left(v, \mu(\sigma), K_{\mu}(\sigma)\right)-f\left(v, \bar{\mu}(\sigma), K_{\bar{\mu}}(\sigma)\right)\right\|
$$

where

$$
\leq \mathbb{L}_{f}\|\mu(\sigma)-\bar{\mu}(\sigma)\|+\mathbb{L}_{f}^{\prime}\left\|K_{\mu}(\sigma)-K_{\bar{\mu}}(\sigma)\right\|,
$$$$
\left\|K_{\mu}(\sigma)-K_{\bar{\mu}}(\sigma)\right\| \leq \frac{\mathbb{L}_{f}\|\mu-\bar{\mu}\|}{1-\mathbb{L}_{f}^{\prime}}
$$

Since $K_{\mu}(v)=f\left(v, \mu(v),{ }_{c}^{\sigma} D^{\alpha} \mu(v)\right)$, therefore,

\section{Similarly, we get}

$$
\left\|K_{\nu}(\sigma)-K_{\bar{\nu}}(\sigma)\right\| \leq \frac{\mathbb{L}_{g}\|\nu-\bar{\nu}\|}{1-\mathbb{L}_{g}^{\prime}} .
$$


Using (31), we get

$$
\begin{aligned}
& \|\mathbb{F}(\mu, \nu)-\mathbb{F}(\bar{\mu}, \bar{\nu})\| \leq a\|\mu-\bar{\mu}\|+\frac{\mathbb{L}_{f} T^{\sigma \alpha}(\|\mu-\bar{\mu}\|)}{\sigma^{\alpha}\left(1-\mathbb{L}_{f}^{\prime}\right) \Gamma(\alpha+1)} \\
& +b\|v-\bar{\nu}\|+\frac{T^{\sigma \alpha} \mathbb{L}_{g}\|\nu-\bar{\nu}\|}{\sigma^{\alpha}\left(1-\mathbb{L}_{g}^{\prime}\right) \Gamma(\alpha+1)} \\
& \leq\left(a+\frac{\mathbb{L}_{f} T^{\sigma \alpha}}{\sigma^{\alpha}\left(1-\mathbb{L}_{f}^{\prime}\right) \Gamma(\alpha+1)}\right)\|\mu-\bar{\mu}\| \\
& +\left(b+\frac{T^{\sigma \alpha} \llbracket_{g}}{\sigma^{\alpha}\left(1-\mathbb{\bigsqcup}_{g}^{\prime}\right) \Gamma(\alpha+1)}\right)\|\nu-\bar{\nu}\| \\
& \leq \gamma_{1}\|\mu-\bar{\mu}\|+\gamma_{2}\|\nu-\bar{\nu}\| \\
& \leq \gamma(\|\mu-\bar{\mu}\|+\|\nu-\bar{\nu}\|) .
\end{aligned}
$$

Here, $\gamma=\max \left\{\gamma_{1}, \gamma_{2}\right\}$, with

$$
\begin{aligned}
& \gamma_{1}=a+\left(\frac{\mathbb{L}_{f} T^{\sigma \alpha}}{\left(\sigma^{\alpha}\left(1-\mathbb{L}_{f}^{\prime}\right) \Gamma(\alpha+1)\right)}\right), \\
& \gamma_{2}=b+\left(\frac{T^{\sigma \alpha} \mathbb{L}_{g}}{\left(\sigma^{\alpha}\left(1-\mathbb{L}_{g}^{\prime}\right) \Gamma(\alpha+1)\right)}\right) .
\end{aligned}
$$

Thus, $\mathbb{F}$ is a contraction. For the continuity and compactness of $\mathbb{G}$, take a sequence $\left\{\mathbb{S}_{n}=\left(\mu_{n}, \nu_{n}\right)\right\}$ in $\mathbb{B}_{r}$ with $\left(\mu_{n}, \nu_{n}\right)$ approaching to $(\mu, \nu)$ as $n$ approaches to $\infty$ in $\mathbb{B}_{r}$. So,

$$
\begin{aligned}
\left\|\mathbb{G}\left(\mu_{n}, v_{n}\right)(\sigma)-\mathbb{G}(\mu, v)(\sigma)\right\|= & \left\|\mathbb{G}_{1}\left(\mu_{n}, v_{n}\right)(\sigma)-\mathbb{G}_{1}(\mu, v)(\sigma)\right\|+\left\|\mathbb{G}_{2}\left(\mu_{n}, v_{n}\right)(\sigma)-\mathbb{G}_{2}(\mu, v)(\sigma)\right\| \\
\leq & \frac{\sigma^{1-\alpha}}{\Gamma(\alpha)} \int_{0}^{\sigma}\left(\sigma^{\sigma}-v^{\sigma}\right)^{\alpha-1} v^{\sigma-1}\left\|\psi\left(v, \mu_{n}(v), v_{n}(v)\right)-\psi(v, \mu(v), v(v))\right\| \mathrm{d} v \\
& +\frac{\sigma^{1-\alpha}}{\Gamma(\alpha)} \int_{0}^{\sigma}\left(\sigma^{\sigma}-v^{\sigma}\right)^{\alpha-1} v^{\sigma-1}\left\|\varphi\left(v, \mu_{n}(v), v_{n}(v)\right)-\varphi(v, \mu(v), v(v))\right\| \mathrm{d} v \\
\leq & \frac{T^{\sigma \alpha}\left(\mathbb{M}_{\psi}\left\|\mu_{n}-\mu\right\|+\mathbb{M}_{\psi}^{\prime}\left\|v_{n}-v\right\|\right)}{\sigma^{\alpha} \Gamma(\alpha+1)}+\frac{T^{\sigma \alpha}\left(\mathbb{M}_{\varphi}\left\|\mu_{n}-\mu\right\|+\mathbb{M}_{\varphi}^{\prime}\left\|v_{n}-v\right\|\right)}{\sigma^{\alpha} \Gamma(\alpha+1)}
\end{aligned}
$$

implies $\left\|\mathbb{G}\left(\mu_{n}, \nu_{n}\right)(\sigma)-\mathbb{G}(\mu, \nu)(\sigma)\right\| \longrightarrow 0$, as $n \longrightarrow \infty$. That is why $\mathbb{G}$ is continuous on $\mathbb{B}_{r}$. Now, for the uniform boundedness of $\mathbb{G}$ on $\mathbb{B}_{r}$, consider $\|\mathbb{G}(\mu, \nu)\|$, and by using (31), we have

$$
\begin{aligned}
\|\mathbb{G}(\mu, \nu)\| & \leq\left\|G_{1}(\mu, \nu)\right\|+\left\|\mathbb{G}_{2}(\mu, \nu)\right\| \\
& \leq \frac{T^{\sigma \alpha}\left(2 l_{\psi}+r\left(l_{\psi}^{1}+l_{\psi}^{2}\right)\right)}{2 \sigma^{\alpha} \Gamma(\alpha+1)}+\frac{T^{\sigma \alpha}\left(2 l_{\varphi}+r\left(l_{\varphi}^{1}+l_{\varphi}^{2}\right)\right)}{2 \sigma^{\alpha} \Gamma(\alpha+1)} \\
& =\left[\frac{T^{\sigma \alpha}\left(l_{\psi}+l_{\varphi}\right)}{r \sigma^{\alpha} \Gamma(\alpha+1)}+\frac{T^{\sigma \alpha}\left(l_{\psi}^{1}+l_{\psi}^{2}+l_{\varphi}^{1}+l_{\varphi}^{2}\right)}{2 \sigma^{\alpha} \Gamma(\alpha+1)}\right] r .
\end{aligned}
$$

Thus, $\mathbb{G}$ is uniformly bounded on $\mathbb{B}_{r}$. Now, for the equicontinuity of the operator $\mathbb{G}$, take $\sigma_{1}, \sigma_{2}$ from $\mathbf{J}$ with $\sigma_{1} \geq \sigma_{2}$ and $(\mu, \nu) \in \mathbb{B}_{r}$. Since $\psi(\sigma, \mu(\sigma), \nu(\sigma))$ is bounded on

$\mathbb{B}_{r}$, we can take $\sup \{\psi(\sigma, \mu(\sigma), \nu(\sigma))\}=M_{0}$. Thus, 
Complexity

7

$$
\begin{aligned}
\left\|\mathbb{G}_{1}(\mu, v)\left(\sigma_{1}\right)-\mathbb{G}_{1}(\mu, v)\left(\sigma_{2}\right)\right\| \leq & \| \frac{\sigma^{1-\alpha}}{\Gamma(\alpha)} \int_{0}^{\sigma_{1}}\left(\sigma_{1}^{\sigma}-v^{\sigma}\right)^{\alpha-1} v^{\sigma-1} \psi(v, \mu(v), v(v)) \\
& -\frac{\sigma^{1-\alpha}}{\Gamma(\alpha)} \int_{0}^{\sigma_{2}}\left(\sigma_{2}^{\sigma}-v^{\sigma}\right)^{\alpha-1} v^{\sigma-1} \psi(v, \mu(v), v(v)) \mathrm{d} v \| \\
\leq & \frac{\sigma^{1-\alpha}}{\Gamma(\alpha)} \int_{0}^{\sigma_{1}}\left(\left(\sigma_{1}^{\sigma}-v^{\sigma}\right)^{\alpha-1}-\left(\sigma_{2}^{\sigma}-v^{\sigma}\right)^{\alpha-1}\right) v^{\sigma-1}\|\psi(v, \mu(v), v(v))\| \mathrm{d} v \\
& +\frac{\sigma^{1-\alpha}}{\Gamma(\alpha)} \int_{\sigma_{1}}^{\sigma_{2}}\left(\sigma_{2}^{\sigma}-v^{\sigma}\right)^{\alpha-1} v^{\sigma-1}\|\psi(v, \mu(v), v(v))\| \mathrm{d} v \\
\leq & M_{0} \frac{\sigma^{1-\alpha}}{\Gamma(\alpha)}\left[\int_{0}^{\sigma_{1}}\left(\left(\sigma_{1}^{\sigma}-v^{\sigma}\right)^{\alpha-1}-\left(\sigma_{2}^{\sigma}-v^{\sigma}\right)^{\alpha-1}\right) v^{\sigma-1} \mathrm{~d} v\right. \\
& \left.+\int_{\sigma_{1}}^{\sigma_{2}}\left(\sigma_{2}^{\sigma}-v^{\sigma}\right)^{\alpha-1} v^{\sigma-1} \mathrm{~d} v\right] \\
= & M_{0} \frac{\sigma^{1-\alpha}}{\Gamma(\alpha)}\left[\frac{\left(\sigma_{2}^{\sigma}-\sigma_{1}^{\sigma}\right)^{\alpha}}{\sigma \alpha}+\frac{\left(\sigma_{2}^{\sigma}-\sigma_{1}^{\sigma}\right)^{\alpha}}{\sigma \alpha}\right] \\
= & \frac{2 M_{0}}{\sigma^{\alpha} \Gamma(\alpha+1)}\left(\sigma_{2}^{\sigma}-\sigma_{1}^{\sigma}\right)^{\alpha}, \text { gives }
\end{aligned}
$$

$\left\|\mathbb{G}_{1}(\mu, \nu)\left(\sigma_{1}\right)-\mathbb{G}_{1}(\mu, \nu)\left(\sigma_{2}\right)\right\| \longrightarrow 0$ as $\sigma_{2} \longrightarrow \sigma_{1}$. Using the same approach with $\sup \{\varphi(\sigma, \mu(\sigma), \nu(\sigma))\}=M_{0}^{\prime}$, we have

$$
\begin{aligned}
\left\|\mathbb{G}_{2}(\mu, \nu)\left(\sigma_{1}\right)-\mathbb{G}_{2}(\mu, \nu)\left(\sigma_{2}\right)\right\|= & \frac{2 M_{0}^{\prime}}{\sigma^{\alpha} \Gamma(\alpha+1)}\left(\sigma_{2}^{\sigma}-\sigma_{1}^{\sigma}\right)^{\alpha} \\
& \longrightarrow 0, \sigma_{1} \longrightarrow \sigma_{2} .
\end{aligned}
$$

Combining these inequalities, we get $\left\|\mathbb{G}(\mu, \nu)\left(\sigma_{1}\right)-\mathbb{G}(\mu, \nu)\left(\sigma_{2}\right)\right\| \longrightarrow 0$ as $\varsigma_{2} \longrightarrow \varsigma_{1}$; hence, $\mathbb{G}$ is relatively compact on $\mathbb{B}_{r}$. Thus, by Arzela-Ascolli (AA) theorem, $\mathbb{G}$ is compact and continuous, so (1) has a unique solution.

Theorem 3. Under conditions $\left(\mathbb{H}_{1}\right)$ to $\left(\mathbb{H}_{5}\right)$ with $\zeta_{f g}<1$, system (1) has a unique solution.

Proof. We define the operator $\Psi=\left(\Psi_{1}, \Psi_{2}\right): \mathbb{X} \times \mathbb{X} \longrightarrow \mathbb{X}$ by

$$
\Psi=\left(\Psi_{1}(\mu, \nu)(\sigma), \Psi_{2}(\mu, \nu)(\sigma)\right), \quad \sigma \in \mathbf{J},
$$

where

$$
\begin{aligned}
\Psi_{1}(\mu, \nu)(\sigma)= & \mu_{0}-h(\mu)+\frac{\sigma^{\alpha-1}}{\Gamma(\alpha)} \int_{0}^{\sigma}\left(\sigma^{\sigma}-v^{\sigma}\right)^{\alpha-1} v^{\sigma-1} f\left(v, \mu(v),{ }_{c}^{\sigma} D^{\alpha} \mu(v)\right) \mathrm{d} v \\
& +\frac{\sigma^{1-\alpha}}{\Gamma(\alpha)} \int_{0}^{\sigma}\left(\sigma^{\sigma}-v^{\sigma}\right)^{\alpha-1} v^{\sigma-1} \psi(v, \mu(v), \nu(v)) \mathrm{d} v, \\
\Psi_{2}(\mu, \nu)(\sigma)= & v_{0}-h(\nu)+\frac{\sigma^{\alpha-1}}{\Gamma(\alpha)} \int_{0}^{\sigma}\left(\sigma^{\sigma}-v^{\sigma}\right)^{\alpha-1} v^{\sigma-1} g\left(v, v(v),{ }_{c}^{\sigma} D^{\alpha} \nu(v)\right) \mathrm{d} v \\
& +\frac{\sigma^{1-\alpha}}{\Gamma(\alpha)} \int_{0}^{\sigma}\left(\sigma^{\sigma}-v^{\sigma}\right)^{\alpha-1} v^{\sigma-1} \psi(v, \mu(v), \nu(v)) \mathrm{d} v .
\end{aligned}
$$


For $(\mu, \nu),(\bar{\mu}, \bar{\nu}) \in \mathbb{X} \times \mathbb{X}$ and $\varsigma \in \mathbf{J}$, we get

$$
\begin{aligned}
& \|\Psi(\mu, \nu)(\sigma)-\Psi(\bar{\mu}, \bar{\nu})(\sigma)\| \leq a\|\mu-\bar{\mu}\|+\frac{T^{\sigma \alpha} \mathbb{L}_{f}\|\mu-\bar{\mu}\|}{\sigma^{\alpha}\left(1-\mathbb{L}_{f}^{\prime}\right) \Gamma(\alpha+1)} \\
& +\frac{T^{\sigma \alpha}\left(\mathbb{M}_{\psi}\|\mu-\bar{\mu}\|+\mathbb{M}_{\psi}^{\prime}\|\nu-\bar{\nu}\|\right)}{\sigma^{\alpha} \Gamma(\alpha+1)} \\
& +b\|\nu-\bar{\nu}\|+\frac{T^{\sigma \alpha} \mathbb{L}_{g}\|\nu-\bar{\nu}\|}{\sigma^{\alpha}\left(1-\mathbb{\mathbb { L }}_{g}^{\prime}\right) \Gamma(\alpha+1)} \\
& +\frac{T^{\sigma \alpha}\left(\mathbb{M}_{\varphi}\|\mu-\bar{\mu}\|+\mathbb{M}^{\prime}\|\nu-\bar{\nu}\|\right)}{\sigma^{\alpha} \Gamma(\alpha+1)} \\
& =\left[a+\frac{T^{\sigma \alpha}}{\sigma^{\alpha} \Gamma(\alpha+1)}\left[\frac{\mathbb{L}_{f}}{1-\mathbb{L}_{f}^{\prime}}+\mathbb{M}_{\psi}+\mathbb{M}_{\varphi}\right]\right]\|\mu-\bar{\mu}\| \\
& +\left[b+\frac{T^{\sigma \alpha}}{\sigma^{\alpha} \Gamma(\alpha+1)}\left[\frac{\mathbb{L}_{g}}{1-\mathbb{L}_{g}^{\prime}}+\mathbb{M}_{\psi}^{\prime}+\mathbb{M}_{\psi}^{\prime}\right]\right]\|\nu-\bar{\nu}\| \\
& \leq \zeta_{f g}(\|\mu-\bar{\mu}\|,\|\nu-\bar{\nu}\|) \text {, with } \\
& \zeta_{f}=a+\frac{T^{\sigma \alpha}}{\sigma^{\alpha} \Gamma(\alpha+1)}\left[\frac{\mathbb{L}_{f}}{1-\mathbb{L}_{f}^{\prime}}+\mathbb{M}_{\psi}+\mathbb{M}_{\varphi}\right] \\
& \zeta_{g}=b+\frac{T^{\sigma \alpha}}{\sigma^{\alpha} \Gamma(\alpha+1)}\left[\frac{\mathbb{L}_{g}}{1-\mathbb{\mathbb { L }}_{g}^{\prime}}+\mathbb{M}_{\psi}^{\prime}+\mathbb{M}_{\psi}^{\prime}\right] \text {, } \\
& \zeta_{f g}=\max \left\{\zeta_{f}, \zeta_{g}\right\} .
\end{aligned}
$$

Hence, $\Psi$ is a contraction, and by the assumption that $\zeta_{f g}<1$, (1) has only one solution.

\section{HU Stability}

Now, we are analyzing different kinds of stabilities such as UU stability of the proposed system given in (1).
Theorem 4. Let the hypothesis from $\left(\mathbb{H}_{1}\right)$ to $\left(\mathbb{H}_{5}\right)$ hold true with the conditions $\zeta_{f g}<1$, and if the matrix $\mathbb{Q} \longrightarrow 0$, then (1) is $\amalg \mathbb{U}$ stable.

Proof. Proceeding from Theorem 3, for any $(\mu, \nu),\left(\mu^{*}, \nu^{*}\right) \in \mathbb{X}$ and $\varsigma \in \mathbf{J}$, we have

$$
\begin{aligned}
\left\|\Psi_{1}(\mu, \nu)(\sigma)-\Psi_{1}\left(\mu^{*}, \nu^{*}\right)(\sigma)\right\| \leq & a\left\|\mu-\mu^{*}\right\|+\frac{T^{\sigma \alpha} \mathbb{L}_{f}\left\|\mu-\mu^{*}\right\|}{\sigma^{\alpha}\left(1-\mathbb{L}_{f}^{\prime}\right) \Gamma(\alpha+1)} \\
& +\frac{T^{\sigma \alpha}\left(\mathbb{M}_{\psi}\left\|\mu-\mu^{*}\right\|+\mathbb{M}_{\psi}^{\prime}\|\nu-\bar{\nu}\|\right)}{\sigma^{\alpha} \Gamma(\alpha+1)} \\
\leq & {\left[a+\frac{T^{\sigma \alpha} \mathbb{L}_{f}}{\sigma^{\alpha}\left(1-\mathbb{L}_{f}^{\prime}\right) \Gamma(\alpha+1)}+\frac{\mathbb{M}_{\psi} T^{\sigma \alpha}}{\sigma^{\alpha} \Gamma(\alpha+1)}\right]\left\|\mu-\mu^{*}\right\| } \\
& +\frac{T^{\sigma \alpha} \mathbb{M}_{\psi}^{\prime}}{\sigma^{\alpha} \Gamma(\alpha+1)}\|v-\bar{\nu}\| \\
= & \mathbb{C}_{1}\left\|\mu-\mu^{*}\right\|+\mathbb{C}_{2}\left\|\nu-\nu^{*}\right\| .
\end{aligned}
$$


Similarly,

$$
\begin{aligned}
& \left\|\Psi_{2}(\mu, \nu)(\sigma)-\Psi_{2}\left(\mu^{*}, v^{*}\right)(\sigma)\right\| \leq \frac{\mathbb{M}_{\varphi} T^{\sigma \alpha}}{\sigma^{\alpha} \Gamma(\alpha+1)}\left\|\mu-\mu^{*}\right\| \\
& +\left[b+\frac{T^{\sigma \alpha} \mathbb{L}_{g}}{\sigma^{\alpha}\left(1-\mathbb{L}_{g}^{\prime}\right) \Gamma(\alpha+1)}+\frac{\mathbb{M}_{\varphi} T^{\sigma \alpha}}{\sigma^{\alpha} \Gamma(\alpha+1)}\right]\left\|\nu-v^{*}\right\| \\
& =\mathbb{C}_{3}\left\|\mu-\mu^{*}\right\|+\mathbb{C}_{4}\left\|\nu-v^{*}\right\|,
\end{aligned}
$$

where

$$
\begin{aligned}
& \mathbb{C}_{1}=a+\frac{T^{\sigma \alpha} \mathbb{L}_{f}}{\sigma^{\alpha}\left(1-\mathbb{L}_{f}^{\prime}\right) \Gamma(\alpha+1)}+\frac{\mathbb{M}_{\psi} T^{\sigma \alpha}}{\sigma^{\alpha} \Gamma(\alpha+1)}, \\
& \mathbb{C}_{2}=\frac{T^{\sigma \alpha} \mathbb{M}_{\psi}^{\prime}}{\sigma^{\alpha} \Gamma(\alpha+1)}, \\
& \mathbb{C}_{3}=\frac{\mathbb{M}_{\varphi} T^{\sigma \alpha}}{\sigma^{\alpha} \Gamma(\alpha+1)}, \\
& \mathbb{C}_{4}=b+\frac{T^{\sigma \alpha} \mathbb{L}_{g}}{\sigma^{\alpha}\left(1-\mathbb{L}_{g}^{\prime}\right) \Gamma(\alpha+1)}+\frac{\mathbb{M}_{\varphi} T^{\sigma \alpha}}{\sigma^{\alpha} \Gamma(\alpha+1)} .
\end{aligned}
$$

Writing together the above inequalities, we have $\left\|\Psi_{1}(\mu, \nu)(\sigma)-\Psi_{1}\left(\mu^{*}, \nu^{*}\right)(\sigma)\right\| \leq \mathbb{C}_{1}\left\|\mu-\mu^{*}\right\|+\mathbb{C}_{2}\left\|\nu-\nu^{*}\right\|$, $\left\|\Psi_{2}(\mu, \nu)(\sigma)-\Psi_{2}\left(\mu^{*}, \nu^{*}\right)(\sigma)\right\| \leq \mathbb{C}_{3}\left\|\mu-\mu^{*}\right\|+\mathbb{C}_{4}\left\|\nu-v^{*}\right\|$.

From this, we get

$$
\left\|\Psi(\mu, v)-\Psi\left(\mu^{*}, v^{*}\right)\right\| \leq \mathbb{Q}\left\|\mu-\mu^{*}, v-v^{*}\right\|,
$$

where $\mathbb{Q}=\left(\begin{array}{ll}\mathbb{C}_{1} & \mathbb{C}_{2} \\ \mathbb{C}_{3} & \mathbb{C}_{4}\end{array}\right)$. As given, $\mathbb{Q}$ converges to 0; therefore, (1) is $\mathbb{U}$ stable.

Example 1. Consider

$$
\begin{aligned}
{ }_{c}^{\sigma} D^{\alpha}(\nu(\sigma))= & \left(\frac{1}{4 e^{\sigma}}+\frac{1}{5 e^{\sigma}}\left(\|\mu(\sigma)\|+\left\|_{c}^{\sigma} D^{\alpha}(\nu(\sigma))\right\|\right)\right) \\
& +\frac{t+\sin |\mu(\sigma)|+\cos |\mu(\sigma)|}{50}, \quad \sigma \in \mathbf{J}, \\
{ }_{c}^{\sigma} D^{\alpha}(v(\sigma))= & \left(\frac{1}{10 e^{2 t}}+\frac{1}{20 e^{\sigma}}\left(\|v(\sigma)\|+\left\|_{c}^{\sigma} D^{\alpha}(v(\sigma))\right\|\right)\right) \\
& +\frac{\cos |\mu(\sigma)|+\|v(\sigma)\|}{t+100} . \\
\mu(0)+\sum_{k=1}^{n} a_{k} \mu\left(\sigma_{k}\right)= & 0, \\
\nu(0)+\sum_{k=1}^{n} b_{k} \nu\left(\sigma_{k}\right)= & 0,
\end{aligned}
$$

where $\sigma=0.4, \alpha \in(0,1), a_{k}, b_{k}>0, k=0,1,2, \ldots, n$. Set functions as

$$
\begin{aligned}
f(\sigma, \mu(\sigma), \nu(\sigma)) & =\frac{1}{4 e^{\sigma}}+\frac{1}{5 e^{\sigma}}\left(\|\mu(\sigma)\|+\left\|_{c}^{\sigma} D^{\alpha}(\nu(\sigma))\right\|\right) \\
g(\sigma, \mu(\sigma), \nu(\sigma)) & =\frac{1}{10 e^{2 t}}+\frac{1}{20 e^{\sigma}}\left(\|v(\sigma)\|+\left\|_{c}^{\sigma} D^{\alpha}(\nu(\sigma))\right\|\right) \\
h(\mu) & =\sum_{k=1}^{n} b_{k} \nu\left(\sigma_{k}\right) \\
h(\nu) & =\sum_{k=1}^{n} b_{k} \nu\left(\sigma_{k}\right) .
\end{aligned}
$$

Taking $(\mu, \nu),(\bar{\mu}, \bar{v})$ from $\mathbb{X}$ and $\sigma \in[0,1]$, we have $\|f(\sigma, \mu(\sigma), \nu(\sigma))-f(\sigma, \bar{\mu}(\sigma), \bar{\nu}(\sigma))\| \leq \frac{1}{5}\|\mu-\bar{\mu}\|$

$$
+\frac{1}{5}\|v-\bar{v}\|
$$

$\|g(\sigma, \mu(\sigma), \nu(\sigma))-g(\sigma, \bar{\mu}(\sigma), \bar{\nu}(\sigma))\| \leq \frac{1}{20}\|\mu-\bar{\mu}\|$

$$
+\frac{1}{20}\|\nu-\bar{\nu}\|
$$

$\|\psi(\sigma, \mu(\sigma), \nu(\sigma))-\psi(\sigma, \bar{\mu}(\sigma), \bar{\nu}(\sigma))\| \leq \frac{1}{50}\|\mu-\bar{\mu}\|$

$$
+\frac{1}{50}\|\nu-\bar{\nu}\|
$$

$\|\varphi(\sigma, \mu(\sigma), \nu(\sigma))-\varphi(\sigma, \bar{\mu}(\sigma), \bar{\nu}(\sigma))\| \leq \frac{1}{100}\|\mu-\bar{\mu}\|$

$$
+\frac{1}{100}\|v-\bar{v}\|
$$

$$
\begin{gathered}
\|h(\mu)-h(\bar{\mu})\| \leq \sum_{k=1}^{m} a_{k}\left\|\mu\left(\sigma_{k}\right)-\bar{\mu}\left(\sigma_{k}\right)\right\|, \\
\|h(\nu)-h(\bar{\nu})\| \leq \sum_{k=1}^{m} b_{k}\left\|\nu\left(\sigma_{k}\right)-\bar{\nu}\left(\sigma_{k}\right)\right\| .
\end{gathered}
$$

In particular, let we take $T=1, \alpha=1 / 2>0, \sigma=0.4$, and from the inequalities in (51), $\mathbb{L}_{f}=\mathbb{L}_{f}^{\prime}=1 / 5, \mathbb{L}_{g}=\mathbb{L}_{g}^{\prime}=$ $1 / 20, \mathbb{M}_{\psi}=\mathbb{M}_{\psi}^{\prime}=1 / 50, \mathbb{M}_{\varphi}=\mathbb{M}_{\varphi}^{\prime}=1 / 100, \quad \sum a_{k}=\sum b_{k}=$ $1 / 3$.

Thus, we have $\zeta_{f g}=0.8328<1$. Therefore, by Theorem 3, (5.1) has a unique solution. After calculations, we get

$$
\mathbb{Q}=\left(\begin{array}{ll}
0.8151 & 0.0357 \\
0.0178 & 0.4450
\end{array}\right) .
$$

Upon calculations, we obtained the eigenvalues 0.81675 and 0.4433 , which show that $\mathbb{Q}$ is converging to zero. Using Theorem 4, the solution of (5.1) is ㅁU stable. 


\section{Conclusion}

In this manuscript, we used $\mathbb{A} \mathbb{A}$ theorem and Banach contraction principle to achieve the sufficient conditions for $\mathbb{E} U$ of solutions to a nonlocal implicit switched system. With the help of assumptions, we proved the U stability result for the couple system given in (1).

\section{Data Availability}

No data were used to support this study.

\section{Conflicts of Interest}

The authors declare that they have no conflicts of interest.

\section{Acknowledgments}

This work was supported by the China Postdoctoral Science Foundation (Grant no. 2019M652348), the Technology Research Foundation of Chongqing Educational Committee (Grant no. KJQN201900539), and the Natural Science Foundation of Chongqing Normal University (Grant no. 16XYY24).

\section{References}

[1] S. Abbas, W. Albarakati, M. Benchohra, and J. J. Nieto, "Global convergence of successive approximations for partial Hadamard integral equations and inclusions," Computers \& Mathematics with Applications, 2016.

[2] D. Baleanu, J. A. T. Machado, and A. C. J. Luo, Fractional Dynamics and Control, Springer, Berlin, Germany, 2012.

[3] M. Benchohra and J. E. Lazreg, "Nonlinear fractional implicit differential equations," Communications in Applied Analysis, vol. 17, pp. 471-482, 2013.

[4] D. B. Dhaigude and S. P. Bhairat, "Existence and uniqueness of solution of cauchy-type problem for Hilfer fractional differential equations," 2017, http://arxiv.org/abs/1704.02174.

[5] A. A. Kilbas, H. M. Srivastava, and J. J. Trujillo, Theory and Applications of Fractional Differential Equations, Elsevier Science, Amsterdam, Netherlands, 2006.

[6] V. Lakshmikantham, S. Leela, and J. V. Devi, Theory of Fractional Dynamic Systems, Cambridge Scientific Publishers, Cambridge, UK, 2009.

[7] I. Podlubny, Fractional Differential Equations, Academic Press, Cambridge, MA, USA, 1999.

[8] S. G. Samko, A. A. Kilbas, and O. I. Marichev, Fractional Integrals and Derivatives: Theory and Applications, Gorden and Breach, Amsterdam, Netherlands, 1987.

[9] K. Diethelm, "The analysis of fractional differential equations," in Lecture Notes in Mathematics, Springer, Berlin, Germany, 2010.

[10] R. Hilfer, Application of Fractional Calculus in Physics, World Scientific, Singapore, 1999.

[11] K. S. Miller and B. Ross, An Introduction to the Fractional Calculus and Differential Equations, John Wiley, Hobooken, NJ, USA, 1993.

[12] Y. Zhou, Basic Theory of Fractional Differential Equations, World Scientific, Singapore, 2014.

[13] Y. Zou and G. He, "On the uniqueness of solutions for a class of fractional differential equations," Applied Mathematics Letters, vol. 74, pp. 68-73, 2017.
[14] Y. Cui, "Uniqueness of solution for boundary value problems for fractional differential equations," Applied Mathematics Letters, vol. 51, pp. 48-54, 2016.

[15] G. Wang, Z. Bai, and L. Zhang, "Successive iterations for unique positive solution of a nonlinear fractional $q$-integral boundary value problem," Journal of Applied Analysis and Computation, vol. 9, no. 4, pp. 1204-1215, 2019.

[16] Y. Zhang, "Existence results for a coupled system of nonlinear fractional multi-point boundary value problems at resonance," Journal of Inequalities and Applications, vol. 2018, p. 198, 2018.

[17] C. Zhai, W. Wang, and H. Li, "A uniqueness method to a new Hadamard fractional differential system with four-point boundary conditions," Journal of Inequalities and Applications, vol. 2018, p. 207, 2018.

[18] F. Wang, Y. Cui, and H. Zhou, "Solvability for an infinite system of fractional order boundary value problems," Annals of Functional Analysis, vol. 10, no. 3, pp. 395-411, 2019.

[19] K. Zhang, J. Wang, and W. Ma, "Solutions for integral boundary value problems of nonlinear Hadamard fractional differential equations," Journal of Function Spaces, vol. 2018, Article ID 2193234, 10 pages, 2018.

[20] K. Zhang and Z. Fu, "Solutions for a class of Hadamard fractional boundary value problems with sign-changing nonlinearity," Journal of Function Spaces, vol. 2019, Article ID 9046472, 7 pages, 2019.

[21] S. M. Ulam, A Collection of the Mathematical Problems, Interscience, New York, NY, USA, 1960.

[22] D. H. Hyers, "On the stability of the linear functional equation," Proceedings of the National Academy of Sciences, vol. 27, no. 4, pp. 222-224, 1941.

[23] T. M. Rassias, "On the stability of the linear mapping in Banach spaces," Proceedings of the American Mathematical Society, vol. 72, no. 2, p. 297, 1978.

[24] D. H. Hyers, G. Isac, and T. M. Rassias, Stability of Functional Equations in Several Variables, Birkhäiuser, Boston, MA, USA, 1998.

[25] S.-M. Jung, "On the hyers-ulam stability of the functional equations that have the quadratic property," Journal of Mathematical Analysis and Applications, vol. 222, no. 1, pp. 126-137, 1998.

[26] S.-M. Jung, "Hyers-Ulam stability of linear differential equations of first order, II," Applied Mathematics Letters, vol. 19, no. 9, pp. 854-858, 2006.

[27] T. Li and A. Zada, "Connections between Hyers-Ulam stability and uniform exponential stability of discrete evolution families of bounded linear operators over Banach spaces," Advances in Difference Equations, vol. 2016, p. 153, 2016.

[28] T. M. Rassias, "On the stability of functional equations and a problem of Ulam," Acta Applicandae Mathematicae, vol. 62, no. 1, pp. 23-130, 2000.

[29] U. Riaz, A. Zada, Z. Ali, M. Ahmad, J. Xu, and Z. Fu, "Analysis of nonlinear coupled systems of impulsive fractional differential equations with Hadamard derivatives," Mathematical Problems in Engineering, vol. 2019, Article ID 5093572, 20 pages, 2019.

[30] U. Riaz, A. Zada, Z. Ali, Y. Cui, and J. Xu, “Analysis of coupled systems of implicit impulsive fractional differential equations involving Hadamard derivatives," Advances in Difference Equations, vol. 2019, p. 226, 2019.

[31] R. Rizwan and A. Zada, "Nonlinear impulsive Langevin equation with mixed derivatives," Mathematical Methods in the Applied Sciences, vol. 43, no. 1, pp. 427-442, 2019. 
[32] R. Rizwan, A. Zada, and X. Wang, "Stability analysis of non linear implicit fractional Langevin equation with non-instantaneous impulses," Advances in Difference Equations, vol. 2019, p. 85, 2019.

[33] S. O. Shah, A. Zada, and A. E. Hamza, "Stability analysis of the first order non-linear impulsive time varying delay dynamic system on time scales," Qualitative Theory of Dynamical Systems, vol. 18, no. 3, pp. 825-840, 2019.

[34] S. O. Shah and A. Zada, "On the stability analysis of nonlinear Hammerstein impulsive integro-dynamic system on time scales with delay," Punjab University Journal of Mathematics, vol. 51, pp. 89-98, 2019.

[35] S. O. Shah and A. Zada, "Existence, uniqueness and stability of solution to mixed integral dynamic systems with instantaneous and noninstantaneous impulses on time scales," Applied Mathematics and Computation, vol. 359, pp. 202-213, 2019.

[36] X. Wang, M. Arif, and A. Zada, “. $\beta$-Hyers-Ulam-Rassias stability of semilinear nonautonomous impulsive system," Symmetry, vol. 231, pp. 1-18, 2019.

[37] J. Wang, A. Zada, and W. Ali, "Ulam's-Type stability of firstorder impulsive differential equations with variable delay in quasi-banach spaces," International Journal of Nonlinear Sciences and Numerical Simulation, vol. 19, no. 5, pp. 553-560, 2018.

[38] J. Wang, A. Zada, and H. Waheed, "Stability analysis of a coupled system of nonlinear implicit fractional anti-periodic boundary value problem," Mathematical Methods in the Applied Sciences, vol. 42, no. 18, pp. 6706-6732, 2019.

[39] A. Zada, W. Ali, and S. Farina, "Hyers-Ulam stability of nonlinear differential equations with fractional integrable impulses," Mathematical Methods in the Applied Sciences, vol. 40, no. 15, pp. 5502-5514, 2017.

[40] A. Zada, W. Ali, and C. Park, "Ulam's type stability of higher order nonlinear delay differential equations via integral inequality of Grönwall-Bellman-Bihari's type," Applied Mathematics and Computation, vol. 350, pp. 60-65, 2019.

[41] A. Zada and A. Mashal, "Stability analysis of nth order nonlinear impulsive differential equations in quasi-banach Space," Numerical Functional Analysis and Optimization, vol. 41, no. 3, pp. 294-321, 2019.

[42] A. Zada and S. O. Shah, "Hyers-Ulam stability of first-order non-linear delay differential equations with fractional integrable impulses," Hacettepe Journal of Mathematics and Statistics, vol. 47, no. 6, pp. 1196-1205, 2018.

[43] A. Zada, O. Shah, and R. Shah, "Hyers-Ulam stability of nonautonomous systems in terms of boundedness of Cauchy problems," Applied Mathematics and Computation, vol. 271, pp. 512-518, 2015.

[44] A. Zada, S. Shaleena, and T. Li, "Stability analysis of higher order nonlinear differential equations in $\beta$-normed spaces," Mathematical Methods in the Applied Sciences, vol. 42, no. 4, pp. 1151-1166, 2019.

[45] M. Obloza, "Hyers stability of the linear differential equation," Rocznik Nauk. Dydakt. Prace Mat.vol. 13, pp. 259-270, 1993.

[46] M. Obloza, "Connections between Hyers and Lyapunov stability of the ordinary differential equations," Rocznik Nauk. Dydakt. Prace Mat.vol. 14, pp. 141-146, 1997.

[47] A. Zada and S. Ali, "Stability analysis of multi-point boundary value problem for sequential fractional differential equations with non-instantaneous impulses," International Journal of Nonlinear Sciences and Numerical Simulation, vol. 19, no. 7-8, pp. 763-774, 2018.
[48] N. Ahmad, Z. Ali, K. Shah, A. Zada, and G. U. Rahman, "Analysis of implicit type nonlinear dynamical problem of impulsive fractional differential equations," Complexity, vol. 2018, Article ID 6423974, 15 pages, 2018.

[49] Z. Ali, A. Zada, and K. Shah, "Existence and stability analysis of three point boundary value problem," International Journal of Applied and Computational Mathematics, vol. 3, no. 1, pp. 651-664, 2017.

[50] A. Ali, B. Samet, K. Shah, and R. A. Khan, "Existence and stability of solution to a toppled systems of differential equations of non-integer order," Boundary Value Problems, vol. 2017, p. 16, 2017.

[51] A. Khan, K. Shah, Y. Li, and T. S. Khan, "Ulam type stability for a coupled system of boundary value problems of nonlinear fractional differential equations," Journal of Function Spaces, vol. 2017, Article ID 3046013, 8 pages, 2017.

[52] J. Wang, K. Shah, and A. Ali, "Existence and Hyers-Ulam stability of fractional nonlinear impulsive switched coupled evolution equations," Mathematical Methods in the Applied Sciences, vol. 41, no. 6, pp. 2392-2402, 2018.

[53] U. N. Katugampola, "New approach to generalized fractional derivatives," Bulletin of Mathematical Analysis and Applications, vol. 6, no. 4, pp. 1-15, 2014.

[54] U. N. Katugampola, "New approach to a generalized fractional integral," Applied Mathematics and Computation, vol. 218, no. 3, pp. 860-865, 2011.

[55] M. Zhang and Y. Liu, "Existence of solutions for implicit fractional differential systems with coupled nonlocal conditions," Advances in Analysis, vol. 2, no. 1, pp. 1-9, 2017.

[56] Z. Bai and H. Lü, "Positive solutions for boundary value problem of nonlinear fractional differential equation," Journal of Mathematical Analysis and Applications, vol. 311, no. 2, pp. 495-505, 2005.

[57] C. Urs, "Coupled fixed point theorems and applications to periodic boundary value problems," Miskolc Mathematical Notes, vol. 14, no. 1, pp. 323-333, 2013. 